\title{
Morphological and Photometric Properties of Active and Non-Active Galaxies in the Green Valley
}

\author{
P. Nkundabakura ${ }^{1}$, A. Mahoro $^{2}$, and M. Pović ${ }^{3}$ \\ ${ }^{1}$ University of Rwanda-College of Education, Fac. science, Physics Department, P.O. Box \\ 5039,Kigali,Rwanda.Email:nkundapheneas@yahoo.fr \\ ${ }^{2}$ Mbarara University of Science and Technology, Fac. of Science, Physics Department, P.O. \\ Box 1410 Mbarara, Uganda. \\ ${ }^{3}$ Institute of Astrophysics of Andalucia (IAA-CSIC), Glorieta de la Astronomía, s/n. E- \\ 18008, Granada, Spain.
}

\begin{abstract}
Context: The differences in properties of active and non-active galaxies in the green valley have been studied in the quest of finding the driving mechanism behind the evolutionary transition from the blue cloud to the red sequence galaxies.

Aims: Using COSMOS data, as one of the deepest surveys, and X-ray detected active galaxies, we studied their properties in the green valley, and established the differences in comparison with the non-active ones.

Methods: Green valley galaxies were selected using the $U-B$ colour criteria of $0.8 \leq U$ - $B \leq 1.2$. We then studied different properties between the two samples such as their morphologies, colours, stellar masses, photometric redshift distributions, and sizes, and performed a statistical comparative study.

Results: We found that in the green valley, active galaxies are dominated by earlier morphological types (elliptical and lenticular galaxies), are more massive, have higher photometric redshifts and redder colours than non-active galaxies.

Conclusions: Our findings suggest that the non-active galaxies in the green valley are different from the active ones, with active galaxies being more evolved.
\end{abstract}

Keywords: galaxies: active, galaxies: fundamental parameters, galaxies: structure, $X$ rays: galaxies

\section{Introduction}

Active galaxies are galaxies that have an Active Galactic Nucleus (AGN) in their centre, which shows a much higher than normal luminosity over a part or over all of the electromagnetic spectrum, especially in X-rays, infrared, and radio. The standard model of AGN suggests that the radiation is a result of accretion of mass by a supermassive black hole at the centre of its host galaxy. AGN are some of the most luminous observed sources, and therefore extremely important for studying the most distant galaxies and the universe in its 
early stage.

Different questions that relate active and non-active galaxies still remain unanswered. Some of them include what is: (1) the effect of AGN on their host galaxy and vice-versa, (2) the origin of the accretion material, (3) the triggering mechanism that converts a normal galaxy into an active one, or (4) the duration of the active phase. A careful selection of samples of active and non-active galaxies, and studying of their properties under the same conditions, could lead to our better understanding on how one galaxy initiate its active phase.

Deep X-ray data provide some of the most complete and unbiased samples of AGN (Mushotzky, 2004; Brandt and Hasinger, 2005). On the other side, morphology and colours are two key parameters used to study the properties of AGN host galaxies, their connection with AGN, and their evolution. It is now well-known from previous studies of colour-magnitude relations that, in gen-eral, normal galaxies may be located in the 'red sequence', populated by massive, bulge-dominated galaxies with older, passively evolving stellar populations, or in the 'blue cloud', populated by blue, star-forming galaxies of small and intermediate masses (e.g., Baldry et al., 2004; Weiner et al., 2005; Cirasuolo et al., 2005; Schawinski et al., 2014). In most of the studies, $\mathrm{X}$-ray detected AGN lie predominantly in the 'green valley' of the colour-magnitude diagrams, a transition region lo-cated between the red sequence and the blue cloud (e.g., Barger et al., 2003; Sánchez et al., 2004; Nandra et al., 2007; Georgakakis et al., 2008; Silverman et al., 2008; Treister et al., 2009; Pović et al., 2012). This has been considered as one of the pieces of evidence of a connection between AGN and galaxy evolution, suggesting that the AGN feedback mechanism may play an important role in quenching star formation, moving the galaxies from the blue cloud to the red-sequence (e.g., Springel et al., 2005; Schawinski et al., 2006; Hasinger, 2008).

However, the real conditions of sources in the green valley and the connection between the normal and active galaxies are still poorly known. In this work, we went deeper in analysing the green valley region, studying the morphological properties, sizes, stellar masses, and colours of its normal and X-ray detected active galaxies, trying to see what are the main similarities and differences between them. For this purpose we used the data from the Cosmic Evolution Survey (COSMOS), having the Hubble Space Telescope (HST) data available in the optical range, ideal for our morphological studies up to higher 
redshifts ( $\mathrm{z}$ 1), and having also the X-ray data avail-able for AGN selection.

The outline of the paper is as follows: In section 2 we present the data used in this study. In section 3 we describe different parameters to be used in the analysis. In section 4 we define the sample of active and nonactive galaxies in the green valley, and we perform statistical comparative analysis of their properties in order to detect whether the two samples have similar or distinct evolutionary tracks. Finally we discuss and summarise the results in section 6 . Throughout the paper we assume a cosmology with $H_{0}=70 \mathrm{~km} / \mathrm{s} / \mathrm{Mpc}, \Omega_{M}=0.3$ and $\Omega_{\Lambda}=0.7$.

\section{Data}

The general sample of galaxies was obtained from the COSMOS deep field survey. COSMOS is a 2 square degree equatorial field survey centred at RA $(\mathrm{J} 2000)=10: 00: 28.6$ and DEC $(\mathrm{J} 2000)=+02: 12: 21.0$ (Scoville et al., 2007). It presents one of the largest and deepest data sets across the entire electromagnetic spectrum, providing the information of about 2 million sources with available high resolution ACS images up to a depth of IAB $\leq 28$ (within $5 \sigma$ ), and $50 \%$ completeness for sources 0.5 " in diameter at $\mathrm{IAB}=26.0$ (Koekemoer et al., 2007). It is a multiwavelength survey, observed with other telescopes, both spacebased (e.g., Spitzer, GALEX, XMM and Chandra) and a number of large ground-based ones (e.g., Subaru, VLA, ESO-VLT, UKIRT, NOAO, and CFHT). It was initially designed to probe, among others, the evolution of galaxies, star formation processes, AGN phenomenology, and largescale structure over the redshift range $0.5 \leq \mathrm{z} \leq 3$.

In the optical, the ACS data were supplemented by observations from the Suprime-Cam on the $8.2 \mathrm{~m}$ Subaru Telescope on Mauna Kea to provide the B -, V -, r' -, i'-, and z' -band photometry. In addition, these data were complemented with further deep imaging in $U$ and $i$ bands from the 3.6 meter optical/infrared Canada-France-Hawaii Telescope (CFHT) in Hawai and in $\mathrm{K}$ band from KPNO/CTIO, UH88, and UKIRT.

In X-rays, COSMOS was observed with XMM-Newton and Chandra telescopes. With XMM-Newton the average exposure time was $\sim 25$ ksec. X-ray source catalogue comprises 1390 different point-like X-ray sources detected over an area of $\sim 2 \operatorname{deg} 2$ (Hasinger et al., 2007; Cappelluti et al., 2007). In this work we used a catalogue of optical counterparts provided by Brusa et al. (2007), with 695 X-ray sources 
detected in the first $1.3 \mathrm{deg} 2$ of the COSMOS XMM-Newton survey, down to limiting fluxes of $10-15$ and $\sim 5 \times 10-15$ erg s-1 cm-2 in the $0.5-2.0 \mathrm{keV}$ and $2.0-10.0 \mathrm{keV}$ bands, respectively. The Chandra COSMOS Survey, with a total exposure time of $1.8 \mathrm{Msec}$, observed the central $0.9 \mathrm{deg} 2$ of the COSMOS field down to limiting fluxes of $1.9 \times$ $10-16$ erg s$-1 \mathrm{~cm}^{-} 2$ and $7.3 \times 10-16$ erg s$-1 \mathrm{~cm}^{-} 2$ in the $0.5-2.0 \mathrm{keV}$ and $2.0-10.0 \mathrm{keV}$ bands, respectively, reaching four times fainter fluxes than XMM-COSMOS. In this work we use the catalogue of Civano et al. (2012) with $1761 \mathrm{X}$-ray point sources with optical counterparts.

\subsection{Sample selection}

Since morphological properties of active and non-active galaxies are crucial for our study, the catalogue where the morphological classification of galaxies is available is our starting point. For this purpose we used the catalogue of Tasca et al. (2011, see section 3 for more information), that provides the morphological classification for 237,912 galaxies with magnitudes $\mathrm{I} \leq 23$. This catalogue is based on the Leauthaud et al. (2007) ACS photometric catalogue which covers a continuous area of $1.64 \mathrm{deg} 2$. To obtain a sample of active galaxies, we cross-matched the Tasca et al. (2011) catalogue with the X-ray ones with available optical counterparts (Civano et al., 2012; Brusa et al., 2007). Since Chandra data are deeper in comparison to XMM-Newton, as pointed above, we combined the two X-ray catalogues by maintaining the sources from Chandra and adding the missing ones detected with XMM-Newton. To select AGN we used the ratio between the X-ray flux on the hard band (2 - $10, \mathrm{keV})$ and optical I band:

$$
\log \mathrm{F}_{X} / \mathrm{F}_{O}=\log \mathrm{F}_{X}+\mathrm{F}_{O} / 2.5+5.352,
$$

and we selected as AGN all sources with $-1 \leq \log \mathrm{F}_{X} / \mathrm{F}_{O} \leq 1$, the condition tested in other works (Alexander et al., 2001; Bauer et al., 2004; Bundy et al., 2007; Trump et al., 2009). Finally, we selected in total a sample of 1,472 AGN sources.

To select a sample of non-active galaxies we excluded all AGN selected sources from the initial Tasca et al. (2011) catalogue. We also excluded sources classified as stars in the Leauthaud et al. (2007) catalogue. In total we selected a sample of 22,154 non-active galaxies. 


\section{Description of available parameters}

In this section we describe the parameters that we used to compare a sample of active and non-active galaxies in the green valley.

- Photometric redshifts.

We used two catalogues of photometric redshifts available in the COSMOS survey. For non-active galaxies we used Ilbert et al. (2009) catalogue, where the photometric redshifts were measured using the Le Phare code (Arnouts et al., 1999; Ilbert et al., 2006), based on $\chi^{2}$ template-fitting procedure. They used the photometric information from 30 broad, intermediate, and narrowband filters from UV, optical, NIR and MIR bands, obtaining an accuracy of $\delta \mathrm{z} /(1+\mathrm{z})=0.007$ for galaxies brighter than $i=22.5$. For a sample of $A G N$ we used the catalogue of Salvato et al. (2011), whose careful measurements were carried out for all Chandra and XMM-Newton detected X-ray sources, using again Le Phare code, including a set of AGN templates, and obtaining the accuracy of $\delta \mathrm{z} /(1+\mathrm{z})=0.015$.

- Absolute magnitudes, rest-frame colours, and stellar masses.

We measured the K-corrections for both, active and non-active samples, by means of the IDL routine KCORRECT (Blanton and Roweis, 2007). We used the photometric information from 10 COSMOS optical and NIR filters and implemented their response files into the code, fitting the SEDs with about 500 available spectral templates (see Blanton and Roweis, 2007, for more information about the templates and SED fitting). We then obtained the K-corrections for all used photometric bands, absolute magnitudes, rest-frame colours, and luminosities of all green valley $\mathrm{AGN}$ and normal galaxies. Moreover, from the KCORRECT SED fits we obtained the stellar masses, using the Chabrier (2003) initial mass function.

- Morphological classification.

As already mentioned, morphological classification of galaxies was obtained from Tasca et al. (2011) catalogue. The catalogue is complete down to $\mathrm{i} \leq 23.0$, and the morphological study was performed on HST/ACS images. Morphological types are estimated using three different automatic, non-parametric methods:

- Class int: based on the concentration index, measuring a fraction of light contained in an inner $30 \%$ flux isophote, and asymmetry,

38

Rwanda Journal, Series D, Volume1, 2016, Life and Natural Sciences: Special issue I 
measuring the degree of symmetry of the galaxy (Abraham et al., 1996; Cassata et al., 2007; Tasca et al., 2011). Late-type galaxies (spirals and irregulars) have lower values of concentration, but higher values of asymmetry in comparison with the early-type galaxies.

- Class_linee: based on 5 morphological parameters, concentration and asymmetry in-dexes, as in previous case, and Gini index, smoothness (or clumpiness), and $M_{20}$ moment of light. The Gini index is also a concentration parameter, presented as the cumulative distribution function of galaxy pixel values (Abraham et al., 2003). The smoothness, quantifies the degree of small scale structure (Conselice et al., 2000). Finally, M20 moment of light is the moment of the brightest $20 \%$ of the galaxy flux.

- Class_svm: based on 7 parameters and obtained through the galSVM code (Huertas-Company et al., 2008), adding the source ellipticity and the surface brightness in the classification in the comparison with the previous method.

In all three methods, galaxies were classified into three classes E/S0 $($ class $=1)$, spirals $($ class $=2)$, and irregulars $($ class $=3)$. We performed visual morphological identification of more than 300 galaxies to test each of the three methods and found that the deviation seems to be the lowest in the case of the class int method. However in order to avoid the subjectivity using the visual methods, the 3 classifications found in the catalogue were used in our comparison (see the next section).

- Sizes.

To quantify the sizes of galaxies we used two different measurements. We extracted the half light radius from Tasca et al. (2011) catalogue. Moreover, we run SExtractor (Bertin and Arnouts, 1996) to obtain the flux radius for different amounts of light, from 5\% up to $100 \%$.

\section{Analysis and Results}

\subsection{Selection of green valley galaxies}

There have been many ways of defining the green valley galaxies, all being based on the bimodal distribution of galaxies when using different colours, e.g. U-B (Nandra et al., 2007), U-V (Moresco et al., 2010), NUV-r (Wyder et al., 2007), or NUV-r+ (Pan et al., 2013). In this work, green valley galaxies were selected using the $\mathrm{U}-\mathrm{B}$ rest-frame colour, and criteria: $0.8 \leq \mathrm{U}-\mathrm{B} \leq 1.2$, as showed in Figure 1, where all galaxies 
with $\mathrm{i} \leq 23$ from the Leauthaud et al. (2007) catalogue are represented. The final sample of green valley galaxies contains 317 AGN and 13,877 normal galaxies, as showed in Table 1 .

\begin{tabular}{|l|l|l|}
\hline & Initial sample & Green valley \\
& & $0.8 \leq \mathrm{U}-\mathrm{B} \leq 1.2$ and $\mathrm{i} \leq 23$ \\
\hline AGN & 1472 & 317 \\
\hline Normal galaxies & 22154 & 13877 \\
& & \\
\hline
\end{tabular}

Table 1: Population of active and non-active galaxies in the initial sample and after the green valley selection.

\subsection{Properties of green valley active and non-active galaxies}

In this section we compared different properties described in section 3 between the samples of AGN and normal galaxies.

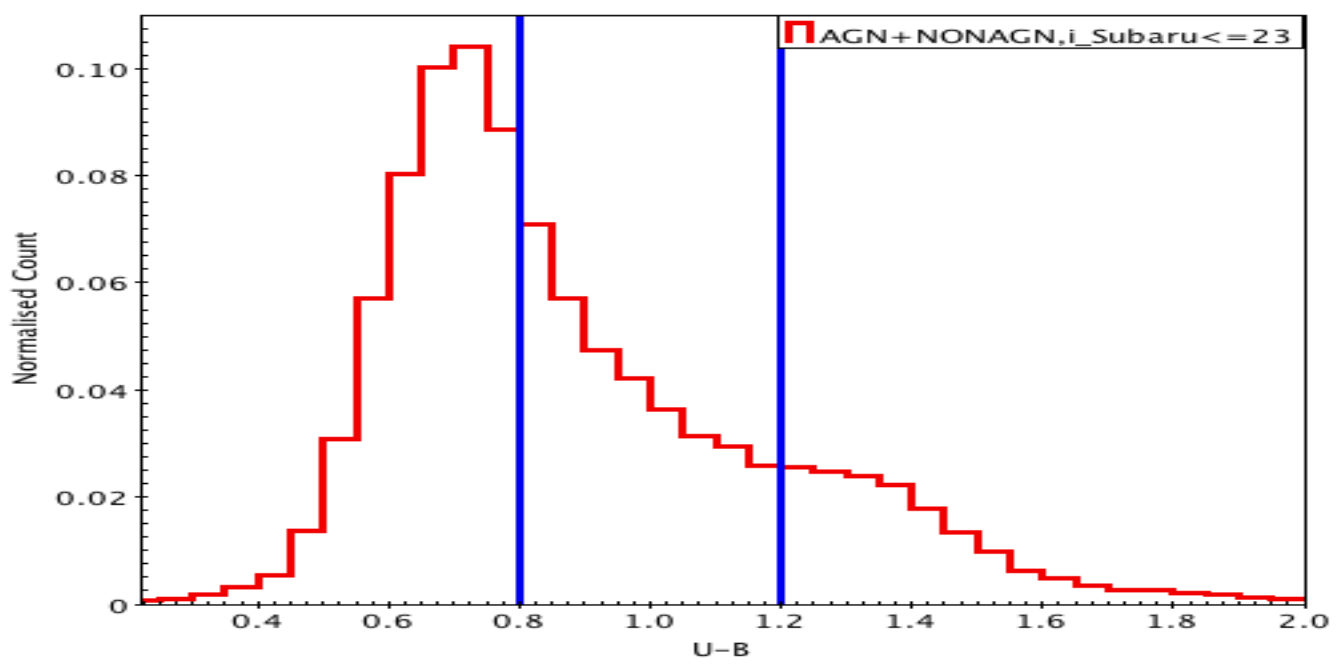

40

Rwanda Journal, Series D, Volume1, 2016, Life and Natural Sciences: Special issue I 
Figure 1: Normalised distribution of U-B colour of the total sample of COSMOS galaxies. Green valley is marked with blue vertical lines.

- Morphological properties.

We compared the morphologies of active and non-active galaxies. Figure 2 shows these comparisons for the three different classification methods, as described above. In general, in all three methods, early-types (E/S0) are dominated by AGN, while late-type galaxies (both spirals and irregulars) are dominated by non-active galaxies. Depending on the classification method used, $40-60 \%$ of AGN and $20-35 \%$ of nonactive galaxies reside in early-types. 

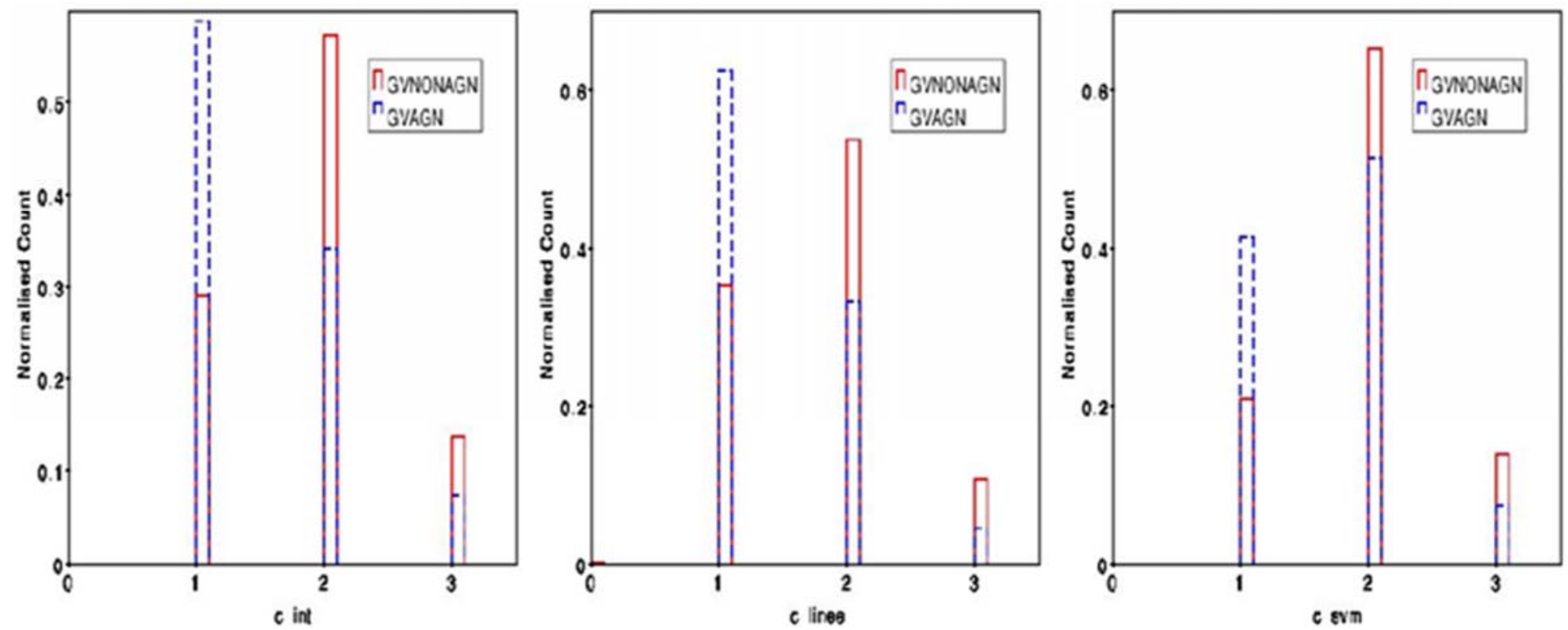

Figure 2: Normalised distributions of morphological types classified by int (left), linee (middle), and galSVM (right) methods (see Sec. 3) of a sample of active (dashed blue line) and non-active (solid red line) galaxies in the green valley. 
- Colour distribution.

Figure 3 shows the normalised distribution of U - B rest-frame colour and comparison between the active and non-active galaxies. The two distributions are significantly different, where redder colours show to be dominated by AGN hosts. $49 \%$ and $36 \%$ of active and non-active galaxies have $\mathrm{U}-\mathrm{B}>1.0$, respectively.

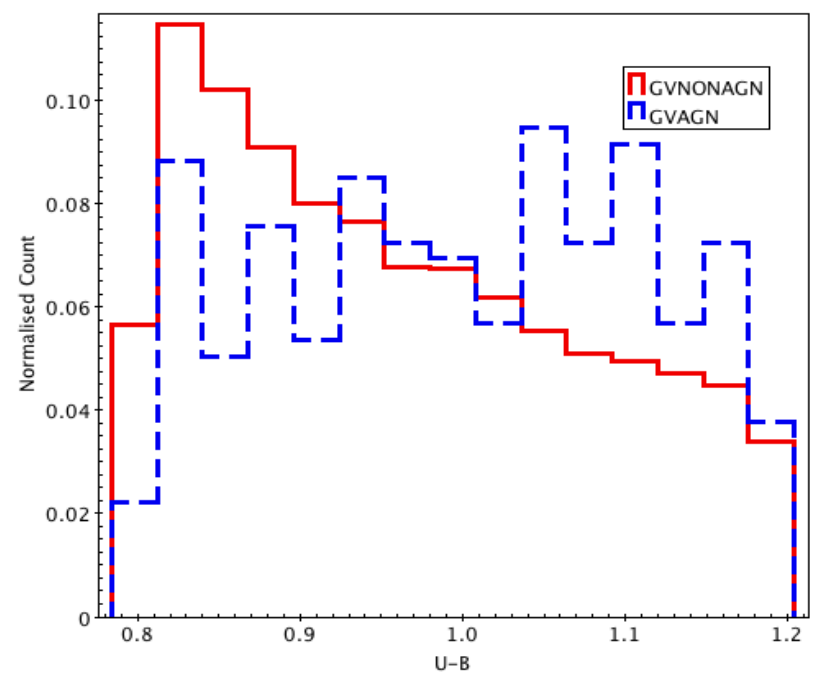

Figure 3: Normalised distributions of $U$ - B rest-frame color of active and non-active galaxies in the green valley. For line descriptions see Fig. 2.

- Photometric redshift distribution

Figure 4 shows the normalised distribution of photometric redshifts for the two samples. Although, the total covered range of photometric $\leq 1.2$, respectively. Galaxies hosted by AGN show higher redshifts with the mean $\mathrm{z}$ of 0.82 , while the mean $\mathrm{z}$ of normal galaxies is 0.59 . 


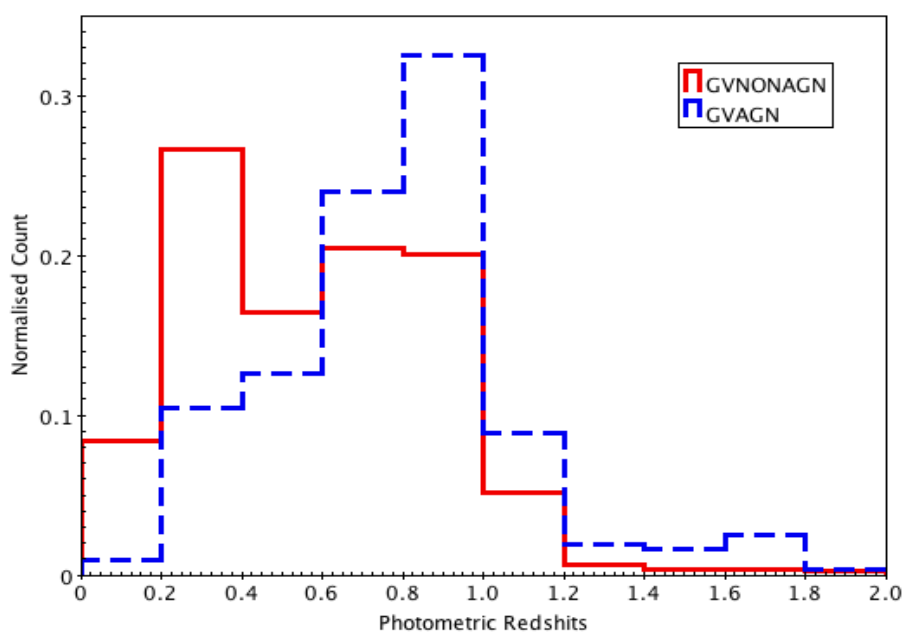

Figure 4: Normalised distributions of photometric redshifts in the green valley. For line descriptions see Fig. 2.

- Stellar masses.

We found significantly different stellar mass distributions of active and non-active green valley galaxies. Figure 5 shows the normalised and normalised cumulative distributions of both samples. AGN hosted galaxies show higher masses than non-active ones, with the mean masses of $3.39 \times 1010 \mathrm{M} \odot$ and $1.17 \times 1010 \mathrm{M} \odot$, respectively. The amount of AGN and normal galaxies with masses above 5x1010 M $\odot$ are $74 \%$ and $34 \%$, respectively. 

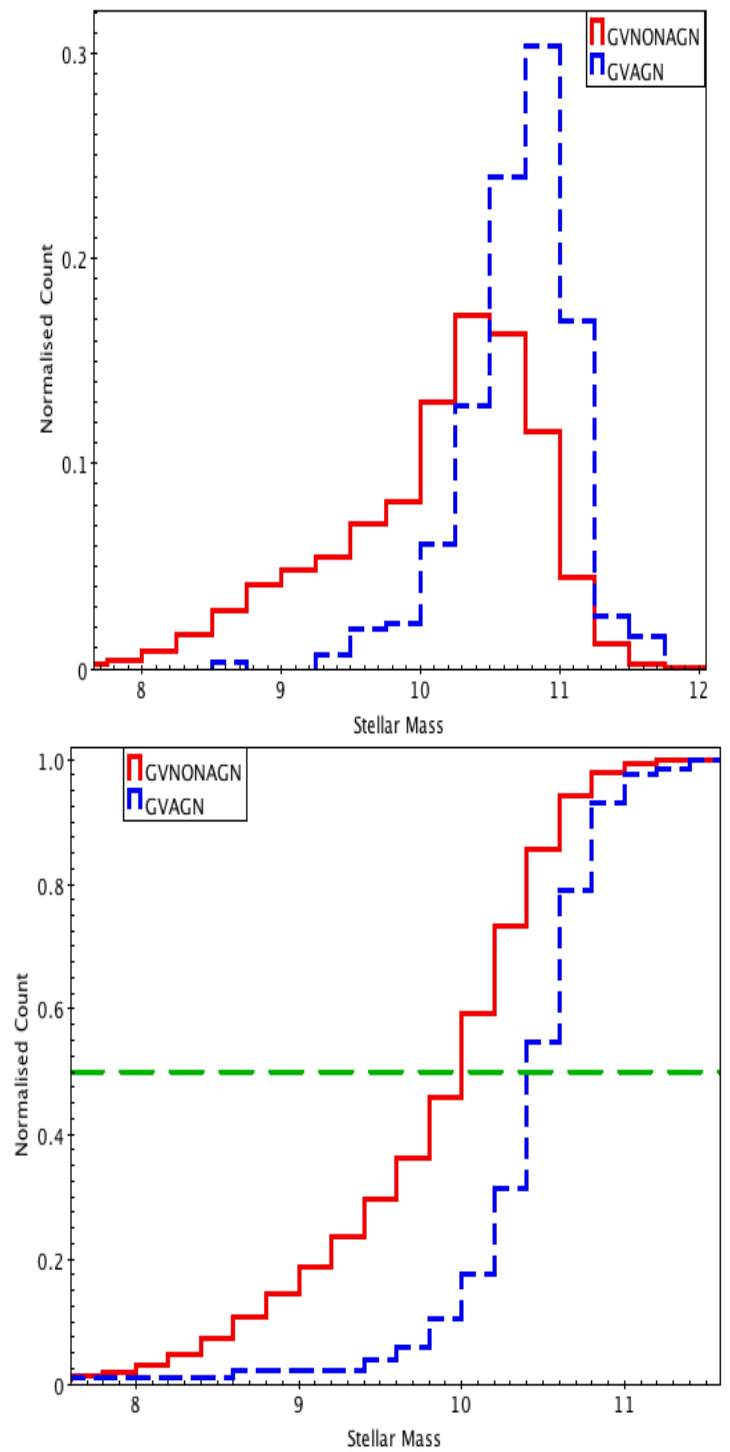

Figure 5: Normalised (left) and normalised cumulative (right) distributions of stellar masses in the Green Valley. For line descriptions see Fig. 2. The horizontal dashed, green line sets the distribution at $50 \%$.

- Sizes.

We compared the sizes at $50 \%$ and $90 \%$ of flux of the two samples of galaxies, as described above, without finding significant differences. Figure 6 shows these comparisons. When comparing the mean values of the distributions they are consistent inside $1 \sigma$. 

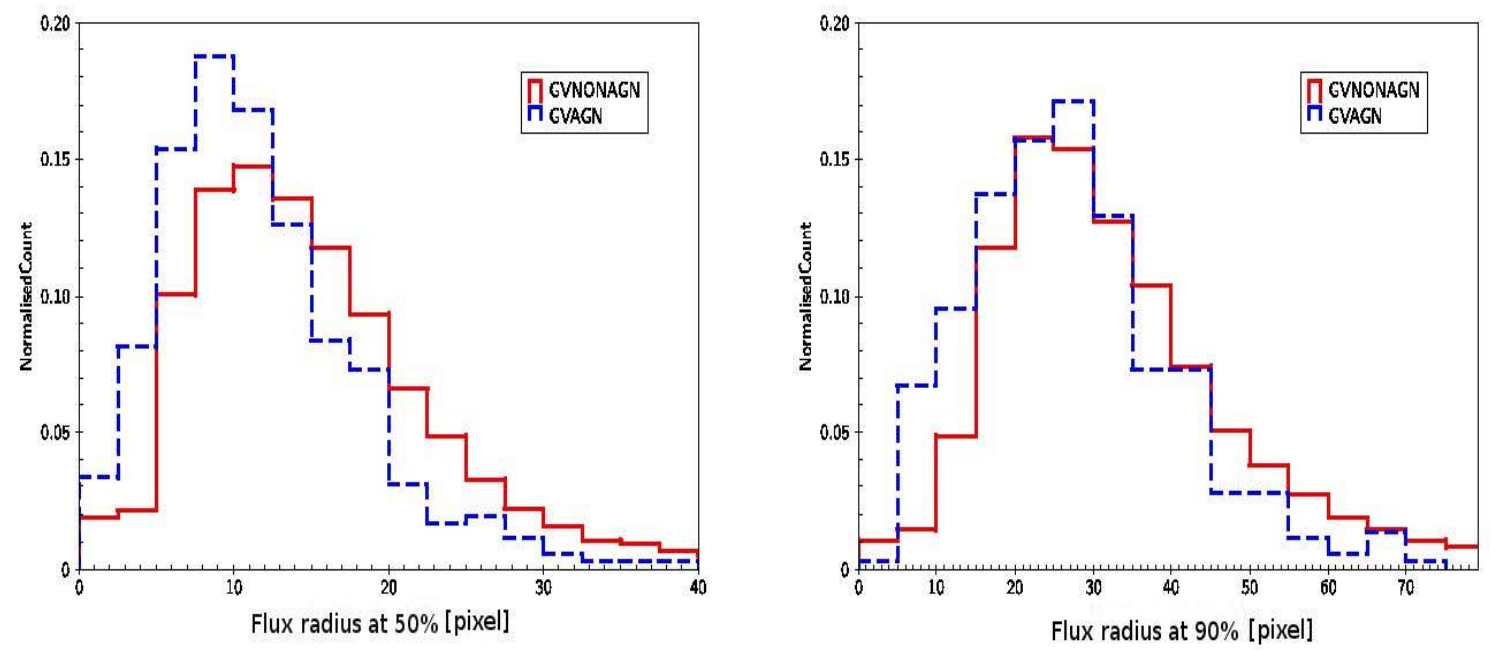

Figure 6: Normalised distributions of flux radius at 50\% (left ) and 90\% (right ) of the flux. For line descriptions see Fig. 2.

\section{Summary}

In this work we compared different properties of active and non-active galaxies that are located in the green valley of the colour-magnitude diagram. Our comparisons suggest that active galaxies seem to be more evolved that the normal ones, being hosted by earlier morphological types, showing redder colours, higher photometric redshifts, and higher stellar masses. Results obtained are consistent with those obtained in the previous studies suggesting that X-ray detected AGN in the green valley may present the transitional population of galaxies between the normal blue cloud and red sequence galaxies.

\section{Acknowledgements}

Financial support from the Swedish International Development Cooperation Agency (Sida) through the International Science Programme (ISP) to University of Rwanda through the Rwanda Astrophysics, Space and Climate Science group is gratefully acknowledged. MP acknowledge financial support from JAE-Doc 
program of the Spanish National Research Council (CSIC), co-funded by the European Social Fund.

\section{References}

Abraham, R. G., et al. (1996). Galaxy morphology to $\mathrm{I}=25 \mathrm{mag}$ in the Hubble Deep Field. Monthly Notices of the Royal Astronomical Society (MNRAS), 279, L47.

Abraham, R. G., van den Bergh, S., and Nair, P. (2003). A New Approach to Galaxy Morphology. I. Analysis of the Sloan Digital Sky Survey Early Data Release. Astrophysical Journal (ApJ), $588,218$.

Alexander, D. M., et al. (2001). The Chandra Deep Field North Survey. VI. The Nature of the Optically Faint X-Ray Source Population. Astronomical Journal (AJ), 122, 2156.

Arnouts, S., et al. (1999). Measuring and modelling the redshift evolution of clustering: the Hubble Deep Field North. Monthly Notices of the Royal Astronomical Society (MNRAS), 310, 540.

Baldry, I. K., et al. (2004). in R. E. Allen, D. V. Nanopoulos, and C. N. Pope (eds.), The New Cosmology: Conference on Strings and Cosmology, Vol. 743 of American Institute of Physics Conference Series, pp 106-119.

Barger, A. J., et al. (2003). Very High Redshift X-Ray-selected Active Galactic Nuclei in the Chandra Deep Field-North. Astrophysical Journal (ApJ), 584, L61. 
Bauer, F. E., et al. (2004). The Fall of Active Galactic Nuclei and the Rise of Star-forming Galaxies: A Close Look at the Chandra Deep Field X-Ray Number Counts. Astronomical Journal (AJ), $128,2048$.

Bertin, E. and Arnouts, S. (1996). SExtractor: Software for source extraction. Astronomy and Astrophysics Suplement Series (A\&AS), 117, 393.

Blanton, M. R. and Roweis, S. (2007). K-Corrections and Filter Transformations in the Ultraviolet, Optical, and Near-Infrared. Astronomical Journal (AJ), 133, 734.

Brandt, W. N. and Hasinger, G. (2005). The Chandra Deep Field North Survey. VI. The Nature of the Optically Faint X-Ray Source Population. Annual Review of Astronomy and Astrophysics $(A \& A S), 43,827$.

Brusa, M., et al. (2007). The XMM-Newton Wide-Field Survey in the COSMOS Field. III. Optical Identification and Multiwavelength Properties of a Large Sample of X-Ray-Selected Sources. Astrophysical Journal Supplement Series (ApJS), 172, 353.

Bundy, K., Treu, T., and Ellis, R. S. (2007). The Mass Assembly History of Spheroidal Galaxies: Did Newly Formed Systems Arise via Major Mergers? Astrophysical Journal (ApJ), 665, L5.

Cappelluti, N., et al. (2007). X-ray source counts in the XMM-COSMOS survey. ArXiv e-prints.

48 
Cassata, P., et al. (2007). The Cosmic Evolution Survey (COSMOS): The Morphological Content and Environmental Dependence of the Galaxy Color-Magnitude Relation at $\mathrm{z} \sim 0.7$. Astrophysical Journal Supplement Series (ApJS), 172, 270.

Chabrier, G. (2003). Galactic Stellar and Substellar Initial Mass Function. Publications of the Astronomical Society of the Pacific (PASP), 115, 763.

Cirasuolo, M., et al. (2005). Dynamical and Photometric Imprints of Feedback Processes on the Formation and Evolution of E/S0 Galaxies. Astrophysical Journal (ApJ), 629, 816.

Civano, F., et al. (2012). The Chandra COSMOS Survey. III. Optical and Infrared Identification of X-Ray Point Sources. Astrophysical Journal Supplement Series (ApJS), 201, 30.

Conselice, C. J., Bershady, M. A., and Jangren, A. (2000). The Asymmetry of Galaxies: Physical Morphology for Nearby and High-Redshift Galaxies. Astrophysical Journal (ApJ), 529, 886.

Georgakakis, A., et al. (2008). X-ray selected AGN in groups at redshifts $\mathrm{z} \sim 1$. Monthly Notices of the Royal Astronomical Society (MNRAS), 391, 183.

Hasinger, G. (2008). Absorption Properties and Evolution of Active Galactic Nuclei. Astronomy and Astrophysics (A\&A), 490, 905.

Hasinger, G., et al. (2007). The XMM-Newton Wide-Field Survey in the COSMOS Field. I. Survey Description. Astrophysical Journal Supplement Series (ApJS), 172, 29. 
Huertas-Company, M., et al. (2008). A robust morphological classification of high-redshift galaxies using support vector machines on seeing limited images. I. Method description. Astronomy and Astrophysics (A\&A), 478, 971.

Ilbert, O., et al.: (2006). Accurate photometric redshifts for the CFHT legacy survey calibrated using the VIMOS VLT deep survey. Astronomy and Astrophysics (A\&A), 457, 841.

Ilbert, O., et al. (2009). Cosmos Photometric Redshifts with 30-Bands for 2-deg2. Astrophysical Journal (ApJ), 690, 1236.

Koekemoer, A. M., et al. (2007). The COSMOS Survey: Hubble Space Telescope Advanced Camera for Surveys Observations and Data Processing. Astrophysical Journal Supplement Series (ApJS), $172,196$.

Leauthaud, A., et al. (2007). Weak Gravitational Lensing with COSMOS: Galaxy Selection and Shape Measurements. Astrophysical Journal Supplement Series (ApJS), 172, 219.

Moresco, M., et al. (2010). zCOSMOS 10k-bright spectroscopic sample. Exploring mass and environment dependence in early-type galaxies. Astronomy and Astrophysics (A\&A), 524, A67.

Mushotzky, R. (2004). in A. J. Barger (ed.), Supermassive Black Holes in the Distant Universe, Vol. 308 of Astrophysics and Space Science Library, p. 53.

50 
Nandra, K., et al. (2007). AEGIS: The Color-Magnitude Relation for XRay-selected Active Galactic Nuclei. Astrophysical Journal (ApJ), 660, L11.

Pan, Z., Kong, X., and Fan, L. (2013). The Spatially Resolved NUV-r Color of Local Star-Forming Galaxies and Clues for Quenching. Astrophysical Journal (ApJ), 776, 14.

Pović, et al. (2012). AGN-host galaxy connection: morphology and colours of X-ray selected AGN at $\mathrm{z} \leq 2$. Astronomy and Astrophysics (A\&A), 541, A118.

Salvato, M., et al. (2011). Dissecting Photometric Redshift for Active Galactic Nucleus Using XMM- and Chandra-COSMOS Samples. Astrophysical Journal (ApJ), 742, 61.

Sánchez, S. F., et al. (2004). Colors of Active Galactic Nucleus Host Galaxies at $0.5<\mathrm{z}<1.1$ from the GEMS Survey. Astrophysical Journal (ApJ), 614, 586.

Schawinski, K., et al. (2006). Suppression of star formation in early-type galaxies by feedback from supermassive black holes. Nature, 442,888 .

Schawinski, et al. (2014). The green valley is a red herring: Galaxy Zoo reveals two evolutionary pathways towards quenching of star formation in early- and late-type galaxies. Monthly Notices of the Royal Astronomical Society (MNRAS), 440, 889 
Scoville, N. (2007). Large Structures and Galaxy Evolution in COSMOS at $\mathrm{z}<1.1$. Astrophysical Journal Supplement Series (ApJS), 172, 1.

Silverman, J. D., et al. (2008). The Evolution of AGN Host Galaxies: From Blue to Red and the Influence of Large-Scale Structures. Astrophysical Journal (ApJ), 675, 1025.

Springel, V., Di Matteo, T., and Hernquist, L. (2005). Black Holes in Galaxy Mergers: The Formation of Red Elliptical Galaxies. Astrophysical Journal (ApJ), 620, L79.

Tasca, L. A. M., et al. (2011). Quantitative morphology of galaxies from the SDSS. I. Luminosity in bulges and discs. Astronomy and Astrophysics (A\&A), 530, 106.

Treister, E., et al. (2009). Optical Spectroscopy of X-ray Sources in the Extended Chandra Deep Field South. Astrophysical Journal (ApJ), 693, 1713.

Trump, J. R., et al. (2009). The COSMOS Active Galactic Nucleus Spectroscopic Survey. I. XMM-Newton Counterparts. Astrophysical Journal (ApJ), 696, 1195.

Weiner, B. J., et al. (2005). The DEEP Groth Strip Galaxy Redshift Survey. III. Redshift Catalog and Properties of Galaxies. Astrophysical Journal (ApJ), 620, 595.

Wyder, T. K., et al. (2007). The UV-Optical Galaxy Color-Magnitude Diagram. I. Basic Properties. Astrophysical Journal Supplement Series (ApJS), 173, 293.

52 